\title{
Native T1 mapping and extracellular volume fraction for differentiation of myocardial diseases from normal CMR controls in routine clinical practice
}

\author{
Rawiwan Thongsongsang ${ }^{1}$, Thammarak Songsangjinda' ${ }^{1}$ Prajak Tanapibunpon ${ }^{2}$ and Rungroj Krittayaphong ${ }^{{ }^{*}}$
}

\begin{abstract}
Background: This study aimed to determine native $T 1$ and extracellular volume fraction (ECV) in distinct types of myocardial disease, including amyloidosis, dilated cardiomyopathy (DCM), hypertrophic cardiomyopathy (HCM), myocarditis and coronary artery disease (CAD), compared to controls.

Methods: We retrospectively enrolled patients with distinct types of myocardial disease, CAD patients, and control group (no known heart disease and negative CMR study) who underwent 3.0 Tesla CMR with routine T1 mapping. The region of interest (ROI) was drawn in the myocardium of the mid left ventricular (LV) short axis slice and at the interventricular septum of mid LV slice. ECV was calculated by actual hematocrit (Hct) and synthetic Hct. T1 mapping and ECV was compared between myocardial disease and controls, and between CAD and controls. Diagnostic yield and cut-off values were assessed.

Results: A total of 1188 patients were enrolled. The average T1 values in the control group were $1304 \pm 42 \mathrm{~ms}$ at septum, and $1294 \pm 37 \mathrm{~ms}$ at mid LV slice. The average T1 values in patients with myocardial disease and CAD were significantly higher than in controls $(1441 \pm 72,1349 \pm 59,1345 \pm 59,1355 \pm 56$, and $1328 \pm 54 \mathrm{~ms}$ for septum of amyloidosis, DCM, HCM, myocarditis, and CAD). Native T1 of the mid LV level and ECV at septum and mid LV with actual and synthetic Hct of patients with myocardial disease or CAD were significantly higher than in controls.

Conclusions: Although native $\mathrm{T} 1$ and $\mathrm{ECV}$ of patients with cardiomyopathy and CAD were significantly higher than controls, the values overlapped. The greatest clinical utilization was found for the amyloidosis group.
\end{abstract}

Keywords: T1 mapping, Extracellular volume fraction, myocardial disease, cardiomyopathy

\section{Introduction}

Cardiac magnetic resonance (CMR) has been increasingly used for the assessment of patients with suspected or known heart disease. Late gadolinium enhancement (LGE) can illustrate myocardial fibrosis. The common

\footnotetext{
*Correspondence: rungroj.kri@mahidol.ac.th

${ }^{1}$ Division of Cardiology, Department of Medicine, Faculty of Medicine

Siriraj Hospital, Mahidol University, 2 Wanglang Road, Bangkoknoi,

Bangkok 10700, Thailand

Full list of author information is available at the end of the article
}

type of fibrosis detected by LGE is replacement fibrosis which happened after myocardial injury [1]. T1 mapping can be performed to demonstrate myocardial tissue characterization. It can detect and quantify infiltrative diseases and diffuse fibrosis [2]. The Society for Cardiovascular Magnetic Resonance and the CMR Working Group of the European Society of Cardiology (ESC) recommended the integration of $\mathrm{T} 1$ mapping into routine CMR practice as a key tissue characterization [3]. Myocardial T1 is prolonged in fibrosis and edema, but 
reduced in lipid accumulation [4]. The extracellular volume fraction (ECV), which represents percentages of extracellular space of the myocardial tissue, can be derived from pre- and post-contrast $\mathrm{T} 1$ values of myocardium and blood pool in combination with hematocrit (Hct) using the established formula [5]. An increased ECV value indicates the presence of excessive collagen deposition or fibrosis, such as in amyloidosis or myocardial infarction [6]. When the actual Hct is unavailable, a synthetic Hct value can be derived from the relationship between Hct and the longitudinal relaxation rate of blood [7]. The ECV is associated with cardiovascular outcomes [8-10]. ECV associated with the all-cause mortality and composite HF endpoints in non-ischemic cardiomyopathy (NICM) patients, using values derived from septal T1 measurements [8], and associated with composite endpoints of hospitalization for heart failure and cardiac death in HFpEF population, using T1 values derived from the whole segment of LV [9]. Meta-analysis of six studies reported that cardiovascular disease patients including NICM, amyloidosis, and small amount of CAD, who have an increase in ECV value had a significantly higher incidence of cardiovascular death and combined cardiac events [10]. The results from previous studies were based on a relatively small sample size and showed inconsistent findings in patients with CAD [11-13].

The primary objective of this study was to determine $\mathrm{T} 1$ values and ECV in distinct types of myocardial disease, including amyloidosis, dilated cardiomyopathy (DCM), hypertrophic cardiomyopathy (HCM), myocarditis, and coronary artery disease (CAD) compared to controls. The secondary objective was to determine the diagnostic yield and cut-off values of T1 and ECV for differentiation of myocardial disease and CAD compared to controls.

\section{Methods}

\section{Patient population}

This study was approved by the Siriraj Institutional Review Board (SIRB) of the Faculty of Medicine Siriraj Hospital, Mahidol University, Bangkok, Thailand. According to committee of SIRB, consent to participate was not required due to the retrospective nature of this study. The study was conducted in compliance with the principles of the Declaration of Helsinki, International Conference on Harmonization Good Clinical Practice guidelines on non-interventional studies. Patients who underwent clinical stress/viability protocol of CMR 3 Tesla with routine T1 mapping at Siriraj Hospital during July 2017 to December 2019 for clinical indication were enrolled. Subjects were categorized based on CMR findings into the myocardial disease group, the CAD group, or the control group. The myocardial disease group included patients diagnosed with amyloidosis, DCM, HCM, or myocarditis.

We used established criteria in combination with CMR criteria for the diagnosis of amyloidosis [14], DCM [15], HCM [16], acute and convalescent myocarditis [17, 18], and CAD [19]. Due to the retrospective nature of this study, patients who had generally accepted contraindications to CMR were not included. Additional exclusion criteria included: (1) artifact of partial volume that was uncorrectable by motion correction program that cause contaminated T1 mapping; (2) moderate or severe valvular heart disease; (3) pericardial disease, congenital heart disease, and other specific cardiomyopathies, such as arrhythmogenic right ventricular cardiomyopathy, restrictive cardiomyopathy, hypertensive cardiomyopathy, stress induced cardiomyopathy, eosinophilic cardiomyopathy, hemochromatosis, and endomyocardial fibrosis; and, (4) patients with acute myocardial infarction.

Figure 1 demonstrates the patient enrollment and group assignment protocol. CAD patients were enrolled at a 1:1 ratio with myocardial disease patients $(n=301$ in each group). The control group comprised patients who did not meet the diagnostic criteria for either myocardial disease or CAD, had no known heart disease, and normal CMR study. The number of patients in the control group was 2 times that of both of the other 2 groups $(n=602)$.

\section{Cardiac magnetic resonance (CMR) protocol}

MR imaging examinations were performed using a commercial Ingenia 3.0T MR system (Philips Medical Systems, Best, the Netherlands). The routine CMR protocol included black blood axial plain images, steady-state free precession (SSFP) cine images of standard long-axis, 2-chamber, 3-chamber, 4-chamber views, and native modified Look-Locker inversion recovery (MOLLI) T1 mapping. Native T1 mapping was performed in middiastole with breath-holding technique and using MOLLI in a single mid-ventricular short axis slice (TR $2.2 \mathrm{~ms}$, TE $1.8 \mathrm{~ms}, 8$ different TIs, matrix $152 \times 150$, field of view $300 \times 300 \mathrm{~mm}^{2}$, flip angle $20^{\circ}$, SENSE 2 , and slice thickness $10 \mathrm{~mm}$ ) [20]. The 5-(3)-3 MOLLI sequence used in this study comprised one inversion pulse with T1 sampling performed over 5 acquisition heartbeats, followed by 3 recovery heartbeats, and a second inversion pulse followed by 3 acquisition heartbeats [20]. Ten minutes after intravenous administration of $0.15 \mathrm{mmol}$ gadolinium-based MRI contrast agent [gadoterate meglumine $\left(\right.$ Dotarem $\left.^{\circledR}\right)$, gadobutrol $\left(\right.$ Gadovist $\left.{ }^{\circledR}\right)$, and gadopentetate dimeglumine (Magnevist ${ }^{\circledR}$ )] per $\mathrm{kg}$ body weight, a T1-weighted Inversion Recovery Fast Low Angle Shot (3D IR-TFE) sequence was acquired in standard long and short axis views (TR $3.4 \mathrm{~ms}$, TE $1.14 \mathrm{~ms}$, matrix 
Screened 2,840 patients

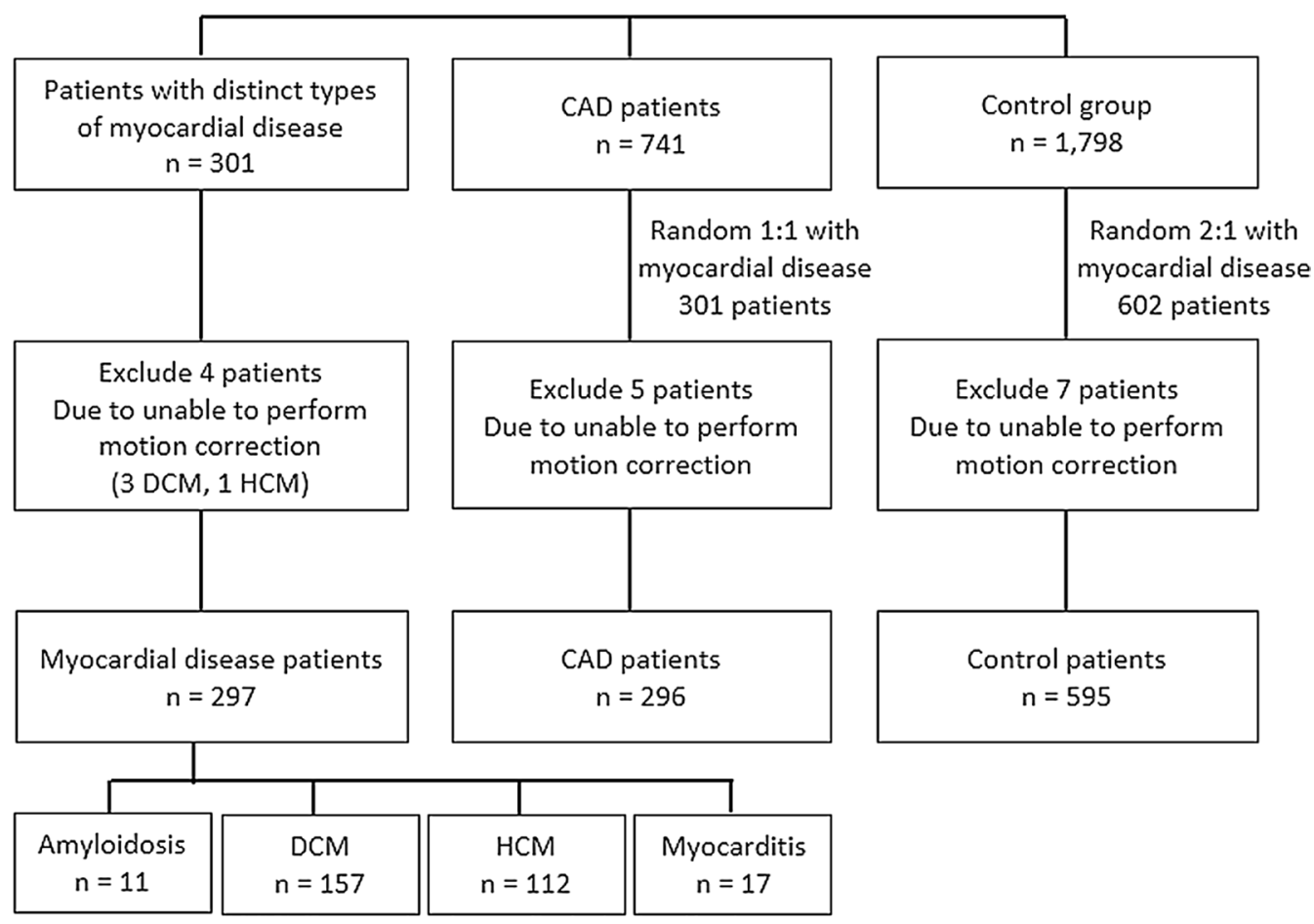

Fig. 1 Details of the study population. CAD coronary artery disease, DCM dilated cardiomyopathy, HCM hypertrophic cardiomyopathy

$152 \times 149$, field of view $270 \times 320$ to $270 \times 380 \mathrm{~mm}^{2}$, flip angle $15^{\circ}$, SENSE 2.5 , and slice thickness $8 \mathrm{~mm}$ ) to assess LGE. Post contrast T1 mapping was performed.

\section{CMR imaging analysis}

All routine CMR analyses were performed using commercially available software (IntelliSpace Portal, version 9; Philips Healthcare, Best, the Netherlands). Routine T1 relaxation maps were also obtained using IntelliSpace Portal (version 9). The region of interest (ROI) was drawn in the myocardium of the mid left ventricular (LV) short axis slice, in the myocardium at the interventricular septum of mid LV slice and the left ventricular blood pool. Native T1 and ECV values were derived from pre- and post-contrast T1 mapping. Greyscale and color mappings were displayed with software default color scheme (Fig. 2A). The mid LV myocardium slice was selected from the region that includes the entire length of the papillary muscles [21]. The ROI was drawn with adequate margins intended to separate myocardium from the area prone to partial volume averaging, such as the area between myocardium and blood. The papillary muscles were excluded as part of the LV myocardium. For the blood pool ROI, care was taken to avoid the papillary muscles. Motion correction was applied when the source images have a different cardiac position between each slice to avoid motion effect on T1 measurement [11, 22, 23]. After the initial image affine registration step of the ROI, the source image was equally subdivided into 6 smaller ROIs to represent mid anterior wall, amid anteroseptal wall, mid inferoseptal wall, mid inferior wall, mid inferolateral wall, and mid anterolateral wall (Fig. 2B). The mid anteroseptal wall and mid inferoseptal wall represent the mid LV septum [21]. Each subdivision underwent another affine transformation to align the features of the target image ROIs with the corresponding ROIs in the source phase image. For CAD patients, the subdivision segment with ischemic-related scar was excluded from ROI of native T1 analysis. However, the subdivision segment with non-ischemic LGE scar was still included $[3,24,25]$. The ECV was calculated using the formula [5]:

$$
\mathrm{ECV}=(1-\text { hematocrit }) \frac{\frac{1}{\text { post contrast T1 myo }}-\frac{1}{\text { native T1 myo }}}{\frac{1}{\text { post contrast T1 blood }}-\frac{1}{\text { native T1 blood }}}
$$

The actual Hct value was determined either on the day of or within 6 months of the CMR study. For 
(See figure on next page.)

Fig. 2 T1 mapping is displayed in color scale. A upper panels, native T1 and T1 enhanced; lower panels, native T1 and T1 enhanced with color scale. B upper panels, T1 map with color scale and region of interest. Mid-left ventricular short axis (Mid LV) native T1 mapping showed endocardial (blue line) and epicardial (red line) contours, as well as the region-of-interest for the calculation of the average myocardial T1 value. Lower panels, the subdivided 6 segments of mid-left ventricular short axis myocardium are represented, as follows: a mid anterior wall, $\mathbf{b}$ mid anteroseptal wall, $\mathbf{c}$ mid inferoseptal wall, $\mathbf{d}$ mid inferior wall, e mid inferolateral wall, and $\mathbf{f}$ mid anterolateral wall

patients without blood sampling, ECV was calculated using a synthetic Hct value that was derived from the relationship between hematocrit and the longitudinal relaxation rate of blood according to the formula: Synthetic Hct $=869.7 \times(1 /$ T1blood $)-0.071$ [7]. This synthetic Hct formula was derived from pre-contrast blood T1 values acquired by MOLLI method on a 3.0 Tesla Philips system (Achieva-dStream, Philips) [7].

\section{Statistical analysis}

Statistical analysis was performed using SPSS Statistics version 18 (SPSS, Inc., Chicago, IL, USA). Normally distributed data are reported as mean plus/minus standard deviation (SD), and categorical data are given as number and percentage. Differences in the average T1 and ECV values between control group and diseased groups were evaluated using independent samples $t$-test. For comparison of two and more than two normally distributed variables, Student's $t$-test and one-way analysis of variance (ANOVA) was used, respectively. Sensitivity, specificity, diagnostic accuracy, positive predictive value (PPV), negative predictive value (NPV), area under the curve (AUC), and cutoff values of native T1 and ECV values to distinguish between controls and the 5 evaluated heart diseases were calculated using receiver operating characteristic (ROC) curve analysis. Comparison of diagnostic yield for detecting diseased cases was performed by the McNemar's test. Comparison of native T1 and ECV between 2 different regions of interest was performed using Pearson's correlation coefficient. Bland-Altman plot, which was used to determine the level of test-retest reliability (calculated as percentage differences from the mean of $1.96 \mathrm{SDs}$ ), was performed using MedCalc statistical software program (MedCalc Software Ltd, Ostend, Belgium). Statistical significance was defined as a $p$-value less than 0.05 .

\section{Results}

We enrolled 2840 patients who underwent routine CMR T1 mapping protocol (Fig. 1). Patients in CAD group were randomly selected to match the number of patients in myocardial disease group. Patients in the control group were also randomized to have the sample size twice the size of the myocardial disease group. The result of that enrollment phase yielded 301 patients in the myocardial disease group, 301 patients in the CAD group, and 602 patients in the control group for a total of 1,204 patients. Sixteen additional patients (4 myocardial disease, 5 CAD, and 7 controls) had to be excluded due to uncorrectable artifacts caused by breathing and/or arrhythmia. A final study population size of 1188 patients were analyzed.

The mean age of patients was $66.7 \pm 13.7$ years, and $48.0 \%$ were males. Patient baseline demographic and clinical characteristics are shown in Table 1. Of the 296 CAD patients, 109 patients had no scar, and 187 patients had scarring on LGE. Three patients had scars in all segments of mid LV myocardium and was all excluded from ROI drawing. Thus, mid LV native T1 value of them could not be derived. There were 49 patients without septum native $\mathrm{T} 1$ value also due to exclusion of scar area in both segments of septum from ROI.

\section{Comparisons of native $\mathrm{T} 1$ and $\mathrm{ECV}$ for discriminating between myocardial disease and controls, and between CAD and controls}

The average native T1 value and ECV are presented in Tables 2 and 3, respectively. Statistical analysis showed significant difference between the control group and the myocardial disease group for all mid LV native T1 values $(p<0.001)$, and for all septum native T1 values $(p<0.001)$. The native T1 values and ECV between myocardial disease and CAD and controls are shown in Fig. 3. Although native $\mathrm{T} 1$ and ECV were significantly higher in cardiomyopathies and CAD compared to controls, there were significant overlaps between cardiomyopathies, CAD and controls except for amyloidosis. The results of ROC curve analysis with corresponding cutoff values of native $\mathrm{T} 1$ for the differentiation of each myocardial disease subgroup and CAD from controls are presented in Table 4.

There were 909 patients who had actual Hct, and their ECV values were calculated using both actual Hct and synthetic Hct. There were 279 patients who did not have actual Hct, so their ECV values were calculated using only synthetic Hct. Statistical analysis showed significant difference between the control group and the myocardial disease group for all mid LV ECV values $(p<0.001)$ and septum ECV values (all $p<0.001$ ), similar to the comparisons for native T1 value. Similar findings were demonstrated for remote area of CAD groups versus controls. The results of ROC curve analysis to evaluate for the 


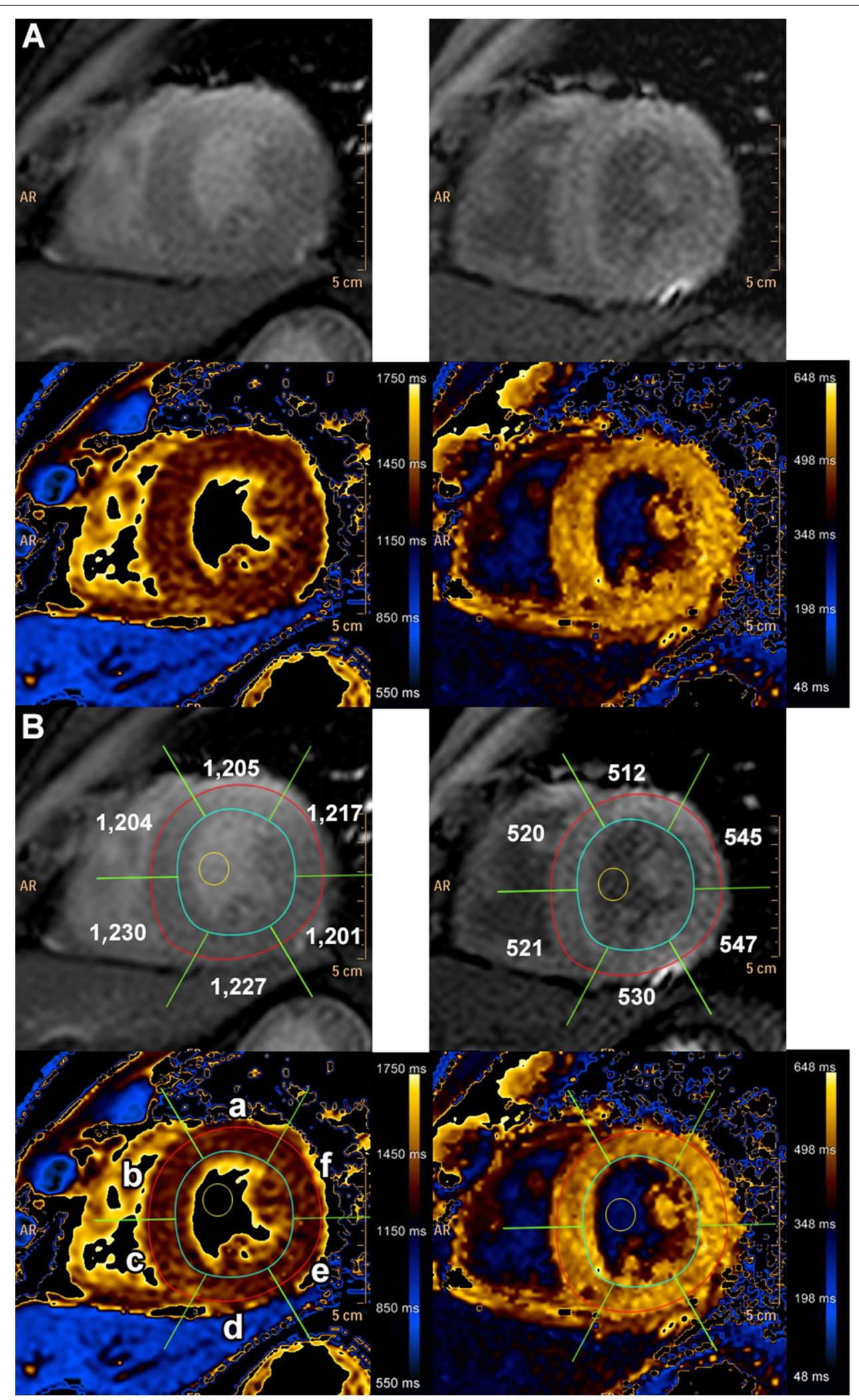


Table 1 Baseline demographic and clinical characteristics

\begin{tabular}{|c|c|c|c|c|c|c|c|}
\hline Characteristics & $\begin{array}{l}\text { All } \\
(N=1188)\end{array}$ & $\begin{array}{l}\text { Amyloidosis } \\
(n=11)\end{array}$ & $\begin{array}{l}\text { DCM } \\
(n=157)\end{array}$ & $\begin{array}{l}\mathrm{HCM} \\
(n=112)\end{array}$ & $\begin{array}{l}\text { Myocarditis } \\
(n=17)\end{array}$ & $\begin{array}{l}\text { CAD } \\
(n=296)\end{array}$ & $\begin{array}{l}\text { Control } \\
(n=595)\end{array}$ \\
\hline Male gender & $570(48.0 \%)$ & $6(54.5 \%)$ & 89 (56.7\%) & $59(52.7 \%)$ & $14(82.4 \%)$ & $198(66.9 \%)$ & 204 (34.3\%) \\
\hline Age, yrs & $66.7 \pm 13.7$ & $57.2 \pm 13.7$ & $59.2 \pm 16.6$ & $64.2 \pm 15.9$ & $54.7 \pm 23.0$ & $71.3 \pm 11.0$ & $67.4 \pm 12.0$ \\
\hline Weight, kg & $65.1 \pm 14.7$ & $61.1 \pm 18.9$ & $64.7 \pm 18.9$ & $64.0 \pm 13.1$ & $65.8 \pm 19.2$ & $64.8 \pm 12.8$ & $65.6 \pm 14.3$ \\
\hline Height, cm & $159.8 \pm 12.6$ & $163.5 \pm 11.5$ & $162.0 \pm 13.2$ & $160.1 \pm 10.8$ & $164.7 \pm 8.6$ & $161.0 \pm 12.5$ & $158.4 \pm 12.7$ \\
\hline $\mathrm{BMI}, \mathrm{ml} / \mathrm{m}^{2}$ & $25.2 \pm 4.8$ & $22.4 \pm 4.3$ & $24.3 \pm 5.4$ & $24.9 \pm 4.3$ & $23.9 \pm 5.4$ & $24.8 \pm 4.4$ & $25.9 \pm 4.9$ \\
\hline Hematocrit, \% & $39.1 \pm 5.3$ & $38.8 \pm 3.2$ & $38.9 \pm 6.9$ & $40.7 \pm 5.0$ & $40.6 \pm 7.3$ & $38.4 \pm 5.6$ & $39.1 \pm 4.7$ \\
\hline $\mathrm{eGFR}, \mathrm{ml} / \mathrm{min} / 1.73 \mathrm{~m}^{2}$ & $70.2 \pm 24.6$ & $88.8 \pm 30.1$ & $71.6 \pm 29.7$ & $66.7 \pm 24.01$ & $78.2 \pm 29.9$ & $64.1 \pm 23.9$ & $74.3 \pm 21.9$ \\
\hline Dyslipidemia & 704 (59.3\%) & $3(27.3 \%)$ & $58(36.9 \%)$ & $58(51.8 \%)$ & $5(29.4 \%)$ & 206 (69.6\%) & $374(62.9 \%)$ \\
\hline Diabetes mellitus & $396(33.3 \%)$ & 1 (9.1\%) & $37(23.6 \%)$ & $28(25.0 \%)$ & $4(23.5 \%)$ & $137(46.3 \%)$ & $189(31.8 \%)$ \\
\hline Hypertension & $786(66.2 \%)$ & $4(36.4 \%)$ & $83(52.9 \%)$ & $67(59.8 \%)$ & $6(35.3 \%)$ & $231(98.0 \%)$ & $395(66.4 \%)$ \\
\hline Heart failure & $161(13.6 \%)$ & $6(54.5 \%)$ & $52(33.1 \%)$ & $13(11.6 \%)$ & $3(17.6 \%)$ & $50(16.9 \%)$ & 37 (6.2\%) \\
\hline
\end{tabular}

Data are presented as number and percentage or mean \pm standard deviation

$B M I$ body mass index, eGFR estimated glomerular filtration rate, $D C M$ dilated cardiomyopathy, $H C M$ hypertrophic cardiomyopathy, $C A D$ coronary artery disease

Table 2 Comparison of native T1 values between patients with myocardial disease or coronary artery disease and control group

\begin{tabular}{|c|c|c|c|c|c|c|c|}
\hline \multirow[t]{2}{*}{ Group } & \multicolumn{3}{|c|}{ Mid LV short-axis native T 1 (ms) } & \multirow[t]{2}{*}{ Group } & \multicolumn{3}{|c|}{ Septum native T1 (ms) } \\
\hline & Mean \pm SD & $95 \% \mathrm{Cl}$ & p-value & & Mean \pm SD & $95 \% \mathrm{Cl}$ & p-value \\
\hline Amyloidosis $(n=11)$ & $1426 \pm 57$ & $1392-1460$ & $<0.001$ & Amyloidosis $(n=11)$ & $1441 \pm 72$ & $1393-1490$ & $<0.001$ \\
\hline $\operatorname{DCM}(n=157)$ & $1341 \pm 52$ & $1333-1349$ & $<0.001$ & $\operatorname{DCM}(n=157)$ & $1349 \pm 59$ & $1340-1358$ & $<0.001$ \\
\hline $\operatorname{HCM}(n=112)$ & $1333 \pm 56$ & $1323-1344$ & $<0.001$ & $\operatorname{HCM}(n=112)$ & $1345 \pm 59$ & $1334-1356$ & $<0.001$ \\
\hline Myocarditis $(n=17)$ & $1344 \pm 62$ & $1315-1374$ & 0.004 & Myocarditis $(n=17)$ & $1355 \pm 56$ & $1327-1384$ & 0.002 \\
\hline $\operatorname{CAD}(n=293)$ & $1316 \pm 51$ & $1310-1322$ & $<0.001$ & $\operatorname{CAD}(n=247)$ & $1328 \pm 54$ & $1321-1334$ & $<0.001$ \\
\hline Control $(n=595)$ & $1294 \pm 37$ & $1291-1297$ & & Control $(n=638)$ & $1304 \pm 42$ & $1301-1308$ & \\
\hline
\end{tabular}

A $p$-value $<0.05$ indicates statistical significance

$S D$ standard deviation, $C l$ confidence interval, DCM dilated cardiomyopathy, HCM hypertrophic cardiomyopathy, $C A D$ coronary artery disease

ability of ECV to differentiate each myocardial disease subgroup and CAD from controls are presented in Table 5. Figure 4 demonstrated ROC curves of native T1 (4 A and $4 \mathrm{~B}$ ) and ECV (4 C to $4 \mathrm{~F}$ ) for the differentiation of cardiomyopathies, CAD compared to controls. The AUCs were in the range of 0.56 to 0.99 with significant $\mathrm{p}$-values. The maximum AUC was for the differentiation of cardiac amyloidosis from controls.

The relation of native T1 (at mid LV) and ECV values (at mid LV using synthetic Hct) among the different groups are demonstrated in Fig. 5. Mid LV was chosen since it might reflect diffuse disease more than septum and ECV using synthetic Hct was chosen to reflect the data of the whole group. Figure 5 which showed 95\% area of each group, demonstrated that the overlapped area of each box were less with the ECV compared to native T1 which indicated that ECV would have an additional value for the differentiation of disease compared to control. To explore whether ECV data have incremental value to native $\mathrm{T} 1$ data alone for the diagnosis of myocardial diseases and $C A D$, we performed additional analysis on comparing diagnostic yield of detecting diseased cases using McNemar's test. The results are shown in Table 6. The results showed that adding ECV to native T1 significantly increased diagnostic sensitivity of DCM and CAD but not significantly increased for amyloidosis, HCM and myocarditis.

proportion of diseased cases that missed diagnosis by using native T1 cut-off. The results showed that the diseases that missed diagnosis by native $\mathrm{T} 1$ can be diagnosed by the cut-off of ECV data using actual Hct in $0 \%$ of amyloidosis, $28.8 \%$ of DCM, $12.2 \%$ of HCM, $25.0 \%$ of myocarditis, and $34.0 \%$ of CAD. If we used cut-off of ECV data using synthetic Hct, the diseases that missed diagnosis by native $\mathrm{T} 1 \mathrm{can}$ be diagnosed in $0 \%$ of amyloidosis, $53.6 \%$ of DCM, $25.0 \%$ of HCM, $10.0 \%$ of myocarditis, and $41.9 \%$ of CAD.

Sensitivity analysis was performed for the assessment of diagnostic yield results that might be influenced by the low prevalence of disease by propensity score matching 


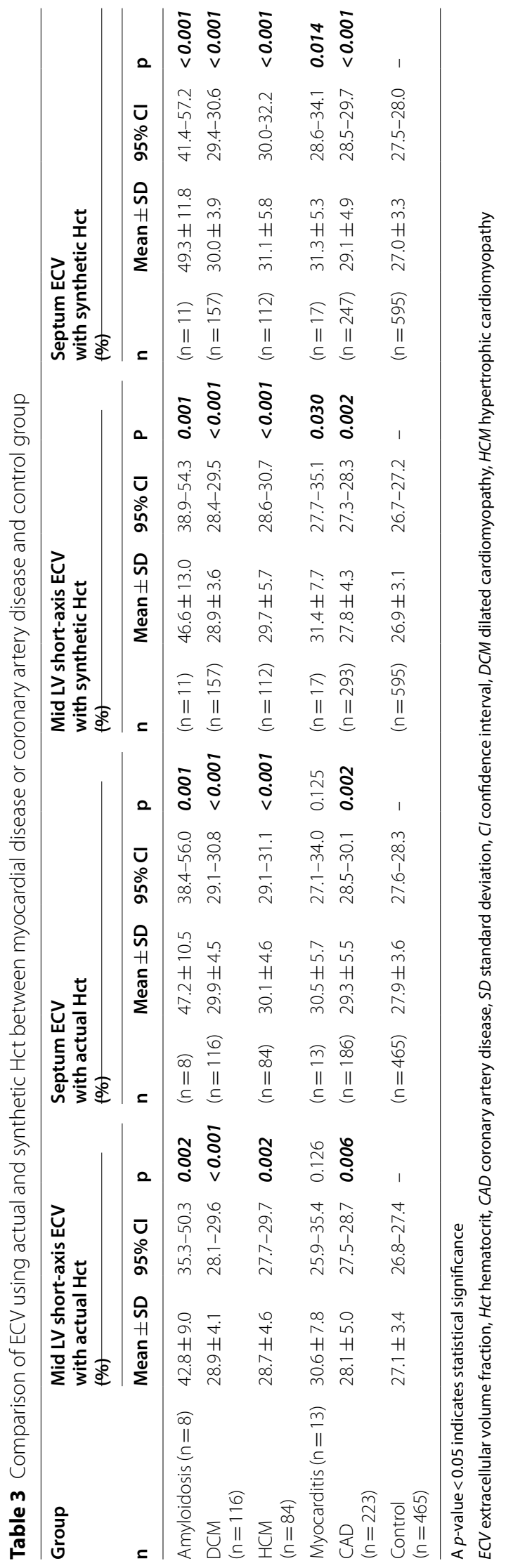



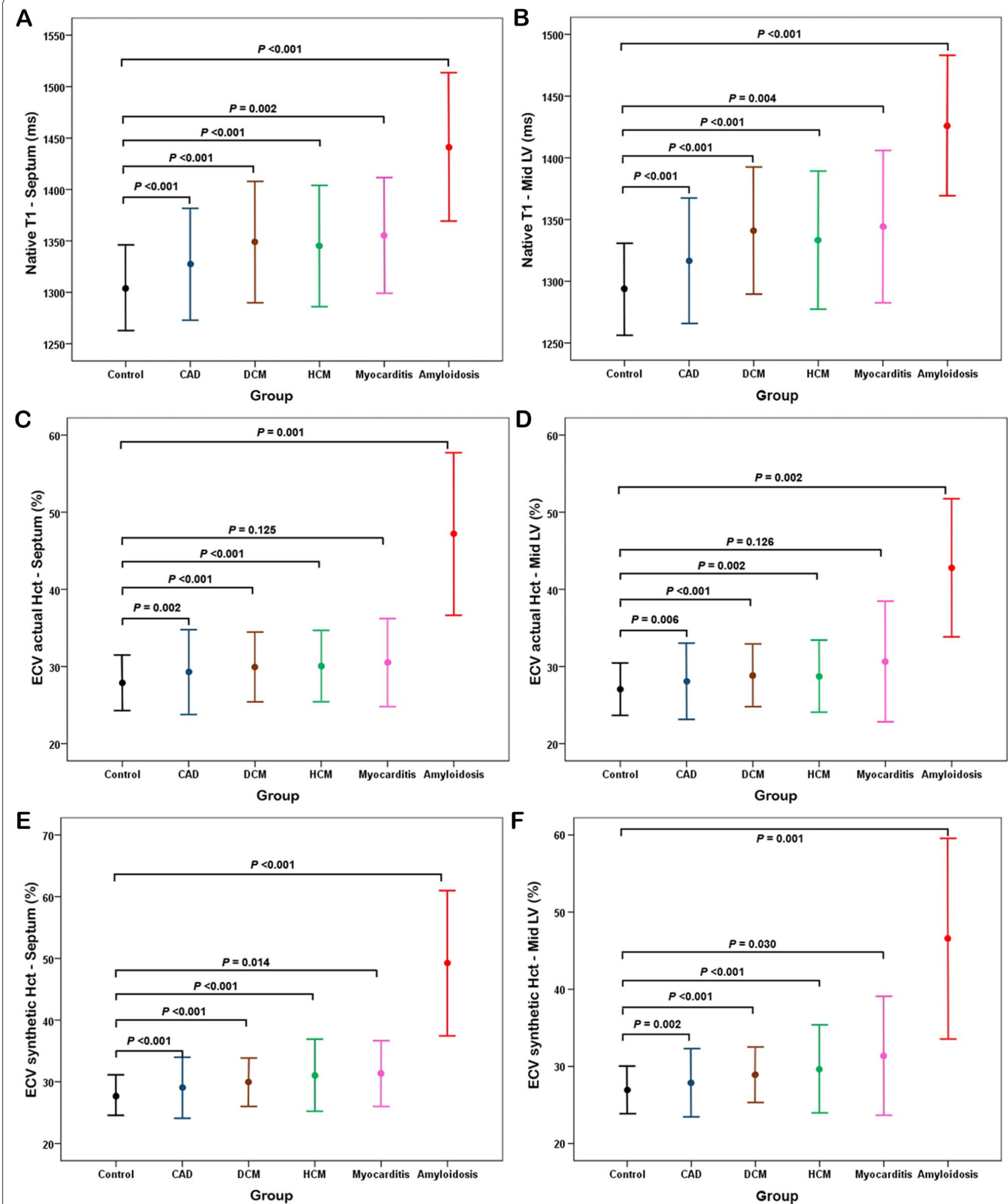

Fig. 3 Comparison of native T1 values and ECV among myocardial diseases, coronary artery disease (CAD) and controls (DCM, dilated cardiomyopathy; HCM, hypertrophic cardiomyopathy). A native T1 at septum, B native T1 at mid LV, C ECV using actual Hct at septum, D ECV using actual Hct at mid LV, E ECV using synthetic Hct at septum, F ECV using synthetic Hct at mid LV 


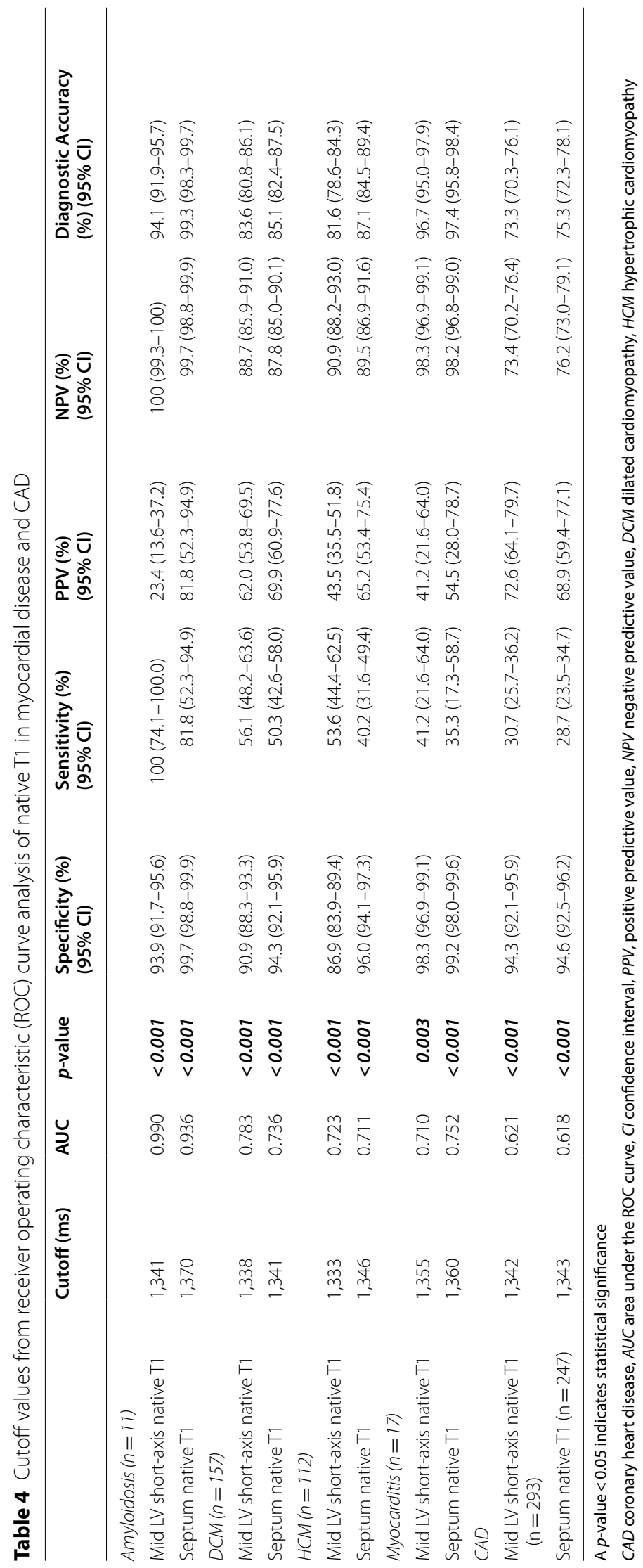


Table 5 Cutoff values from receiver operating characteristic (ROC) curve analysis of mid LV short-axis ECV in myocardial disease and CAD

\begin{tabular}{|c|c|c|c|c|c|c|c|c|}
\hline & Cutoff (\%) & AUC & $p$-value & $\begin{array}{l}\text { Specificity (\%) } \\
(95 \% \mathrm{Cl})\end{array}$ & $\begin{array}{l}\text { Sensitivity (\%) } \\
(95 \% \mathrm{Cl})\end{array}$ & $\begin{array}{l}\text { PPV (\%) } \\
(95 \% \mathrm{Cl})\end{array}$ & $\begin{array}{l}\text { NPV (\%) } \\
(95 \% \mathrm{CI})\end{array}$ & $\begin{array}{l}\text { Diagnostic Accuracy } \\
(\%)(95 \% \mathrm{Cl})\end{array}$ \\
\hline \multicolumn{9}{|l|}{ Amyloidosis } \\
\hline $\begin{array}{l}\text { Mid LV short-axis actual } \\
\text { ECV }(n=8)\end{array}$ & 40.0 & 0.882 & $<0.001$ & $99.6(98.4-99.9)$ & $87.5(52.9-97.8)$ & $77.8(45.3-93.7)$ & $99.8(98.8-100)$ & 99.4 (98.2-99.8) \\
\hline $\begin{array}{l}\text { Mid LV short-axis syn- } \\
\text { thetic ECV }(n=11)\end{array}$ & 32.6 & 0.910 & $<0.001$ & $97.6(96.1-98.6)$ & $81.8(52.3-94.9)$ & $39.1(22.2-59.2)$ & $99.7(98.8-99.9)$ & $97.4(95.8-98.4)$ \\
\hline \multicolumn{9}{|l|}{$D C M$} \\
\hline $\begin{array}{l}\text { Mid LV short-axis actual } \\
\text { ECV }(n=116)\end{array}$ & 28.8 & 0.638 & $<0.001$ & $72.9(68.7-76.7)$ & $51.7(42.7-60.6)$ & $32.3(26.0-39.3)$ & $85.8(82.0-88.9)$ & $68.7(64.8-72.3)$ \\
\hline $\begin{array}{l}\text { Mid LV short-axis syn- } \\
\text { thetic ECV }(n=157)\end{array}$ & 27.0 & 0.675 & $<0.001$ & $55.6(51.6-59.6)$ & $72.6(65.2-79.0)$ & $30.2(25.8-35.0)$ & 88.5 (84.9-91.4) & $59.2(55.6-62.6)$ \\
\hline \multicolumn{9}{|l|}{ HCM } \\
\hline $\begin{array}{l}\text { Mid LV short-axis actual } \\
\text { ECV }(n=84)\end{array}$ & 31.8 & 0.601 & 0.003 & $93.8(91.2-95.6)$ & $25.0(17.0-35.2)$ & $42.0(29.4-55.8)$ & $87.4(84.2-90.0)$ & $83.2(79.9-86.1)$ \\
\hline $\begin{array}{l}\text { Mid LV short-axis syn- } \\
\text { thetic ECV }(n=112)\end{array}$ & 29.7 & 0.670 & $<0.001$ & $83.7(80.5-86.4)$ & $46.4(37.5-55.6)$ & $34.9(27.7-42.8)$ & $89.2(86.4-91.6)$ & $77.8(74.6-80.7)$ \\
\hline \multicolumn{9}{|l|}{ Myocarditis } \\
\hline $\begin{array}{l}\text { Mid LV short-axis actual } \\
\text { ECV }(n=13)\end{array}$ & 30.5 & 0.625 & 0.124 & $85.4(81.9-88.3)$ & $53.8(29.1-76.8)$ & $9.3(4.6-18.0)$ & 98.5 (96.8-99.3) & $84.5(81.0-87.5)$ \\
\hline $\begin{array}{l}\text { Mid LV short-axis syn- } \\
\text { thetic ECV }(n=17)\end{array}$ & 31.3 & 0.694 & 0.006 & $92.9(90.6-94.7)$ & $41.2(21.6-64.0)$ & $14.3(7.1-26.7)$ & $98.2(96.8-99.0)$ & $91.5(89.0-93.5)$ \\
\hline \multicolumn{9}{|l|}{$C A D$} \\
\hline $\begin{array}{l}\text { Mid LV short-axis actual } \\
\text { ECV }(n=223)\end{array}$ & 27.9 & 0.559 & 0.012 & $65.4(60.9-69.6)$ & $49.3(42.8-55.8)$ & $40.6(34.9-46.5)$ & $72.9(68.4-76.9)$ & $60.2(56.5-63.8)$ \\
\hline $\begin{array}{l}\text { Mid LV short-axis syn- } \\
\text { thetic ECV }(n=293)\end{array}$ & 27.3 & 0.561 & 0.003 & $58.5(54.5-62.4)$ & $53.9(48.2-59.5)$ & $39.0(34.4-43.8)$ & $72.0(67.9-75.9)$ & $57.0(53.7-60.2)$ \\
\hline
\end{tabular}

A $p$-value $<0.05$ indicates statistical significance

$C A D$ coronary heart disease, $A U C$ area under the ROC curve, $C l$ confidence interval, $P P V$ positive predictive value, NPV negative predictive value, $E C V$ extracellular volume fraction, DCM dilated cardiomyopathy, HCM hypertrophic cardiomyopathy

of case and control at 1:1 ratio. Cases and controls were matched for age, gender, clinical presentation, and cardiovascular risk factors. The results of native T1 and ECV are shown in Additional files 1 and 2. The PPV increased whereas the NPV decreased.

\section{Correlation and agreement for native T1 and ECV compared between different measurement approaches}

The average native T1 compared between different measurement approaches (ROI drawn in mid LV myocardium and septum) showed a statistically significant correlation $(\mathrm{R}=0.924, p<0.001)$. The calculated ECV compared between mid LV and septum native T1 when using actual Hct showed a statistically significant correlation ( $\mathrm{R}=0.944, p<0.001)$, and significant correlation was also found for ECV by synthetic Hct $(R=0.944$, $p<0.001)$. The calculated mid LV ECV by actual Hct and synthetic Hct showed statistically significant correlation $(\mathrm{R}=0.837, p<0.001)$, and a significant correlation of both approaches was also found for septum ECV $(R=0.847$, $p<0.001$ ).

The Bland-Altman plots confirmed the good agreement between septal and mid-LV T1 measurements and between ECV derived from the actual and synthetic Hct (Fig. 6).

\section{Discussion}

Native T1 of myocardium was significantly higher in the myocardial disease and CAD groups compared to the control group. This study showed that myocardial disease and CAD can be differentiated from control group by means of native T1 mapping with good diagnostic accuracy. Amyloidosis had the highest AUC, sensitivity and specificity.

For differentiation between amyloidosis and controls the native $\mathrm{T} 1$ at septum and mid LV had a high diagnostic accuracy but the PPV of native T1 at mid LV was relatively low which may be related to the small number of disease subgroups. Karamitsos, et al. reported elevations 

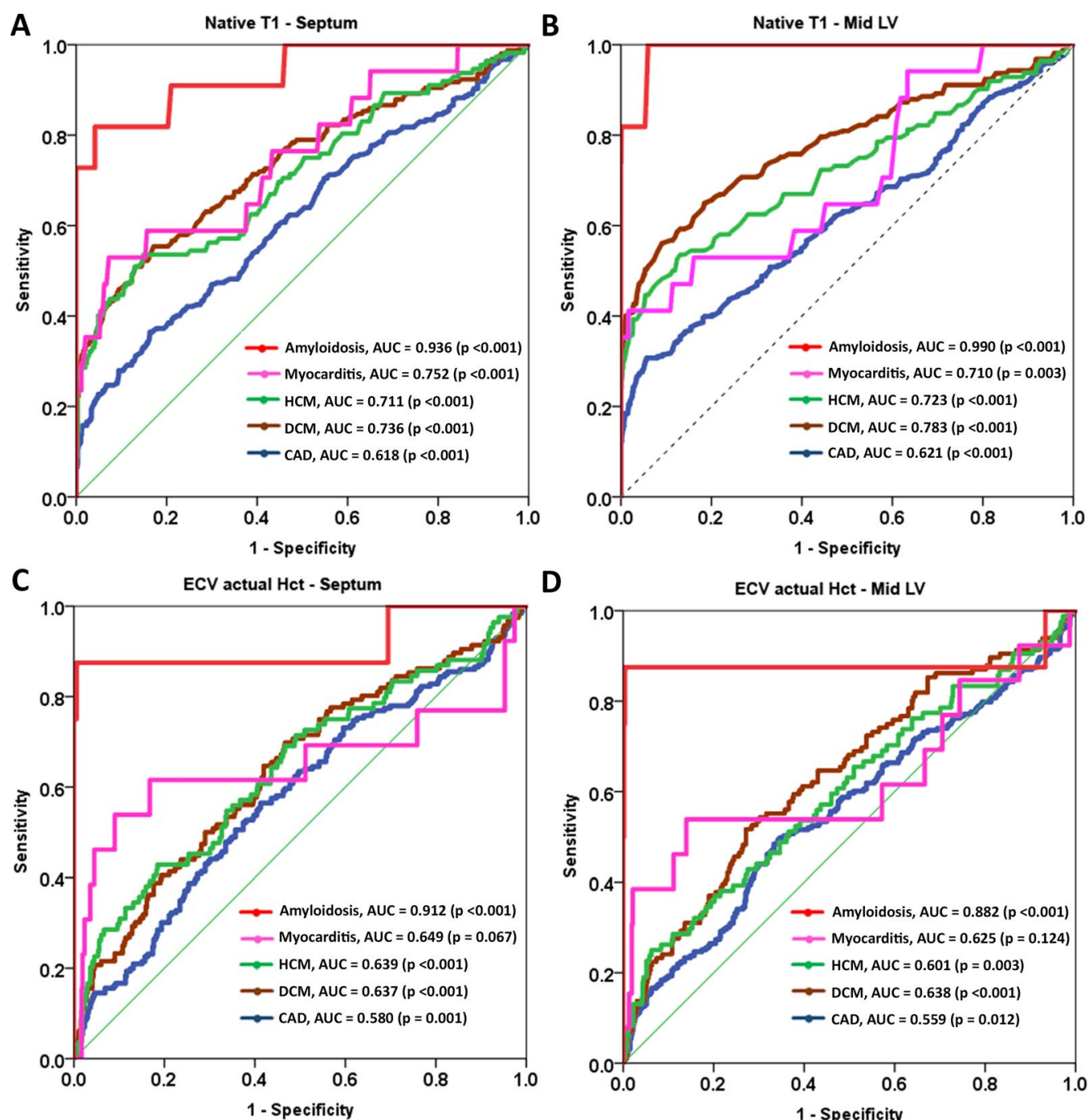

D
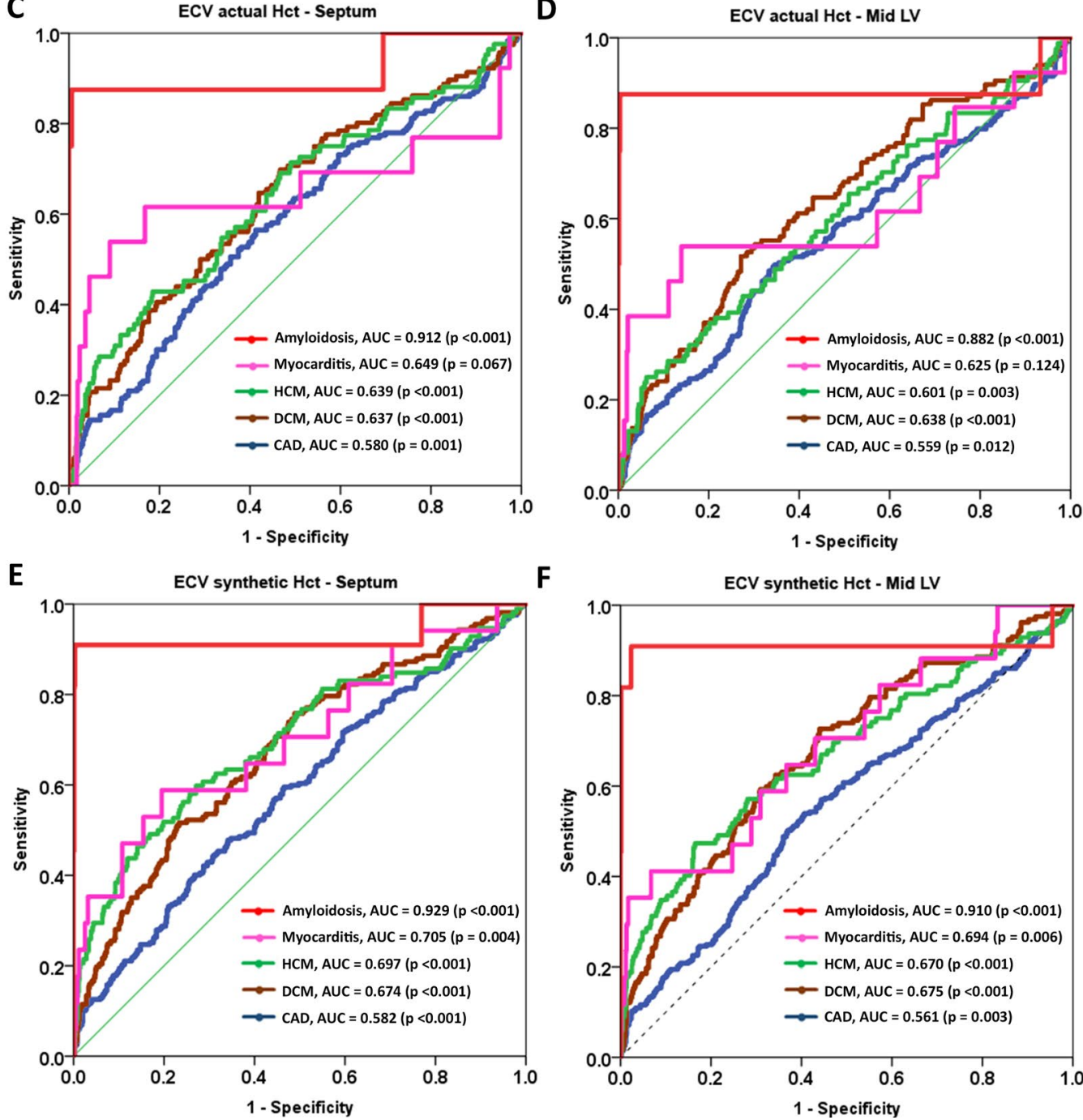

Fig. 4 Receiver operating characteristic curve of native T1 and ECV for the diagnosis of amyloidosis, myocarditis, hypertrophic cardiomyopathy (HCM), dilated cardiomyopathy (DCM), and coronary artery disease (CAD). AUC = area under the curve. A Native T1-septum, B Native T1-mid LV, C ECV actual Hct-septum, D ECV actual Hct-mid LV, E ECV synthetic Hct-septum and $\mathbf{F}$ ECV synthetic Hct-mid LV 


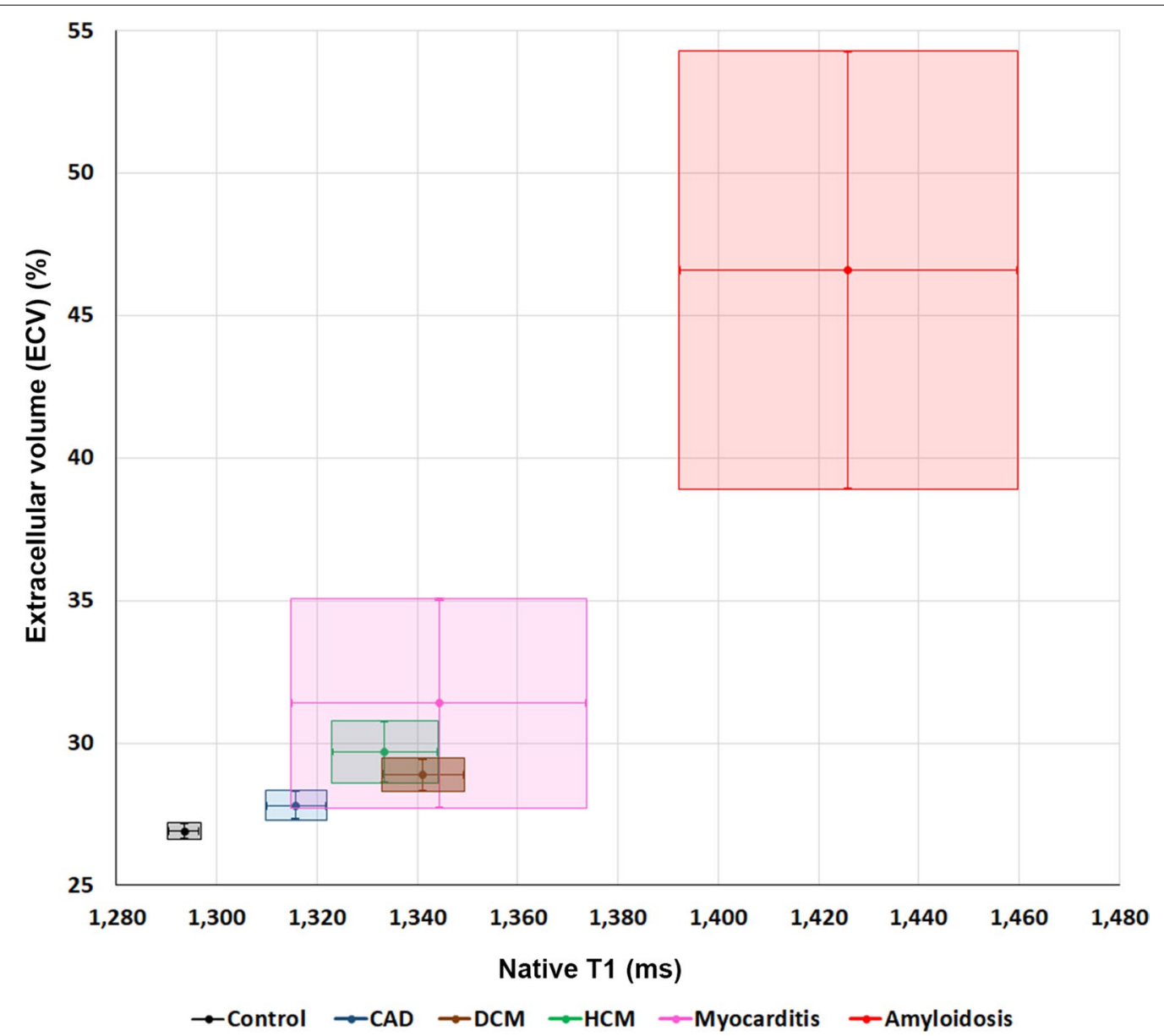

Fig. 5 95\% Confidence interval (Cl) box graph showing comparison of native T1 at mid LV and extracellular volume (ECV) using synthetic Hct at mid LV in the myocardial diseases, coronary artery disease (CAD), and control groups (DCM, dilated cardiomyopathy; HCM, hypertrophic cardiomyopathy)(Horizontal dimension of each box indicates $95 \% \mathrm{Cl}$ of native $\mathrm{T1}$, vertical dimension indicates $95 \% \mathrm{Cl}$ of ECV, mid-point indicates mean value of native $T 1$ and $E C V$ )

Table 6 Sensitivity of native T1, ECV using synthetic hematocrit at mid LV and combination (native T1 followed by ECV if T1 was normal) in the disgnosis of amyloidosis, dilated cardiomyopathy (DCM0, hypertrophic cardiomyopathy (HCM), myocarditis, and coronary artery disease (CAD). P-values of comparison of combination of test and single test based on McNemar's test are shown

\begin{tabular}{|c|c|c|c|c|c|c|}
\hline Test & & $\begin{array}{l}\text { Amyloidosis } \\
(n=11)\end{array}$ & $\begin{array}{l}\text { DCM } \\
(n=157)\end{array}$ & $\begin{array}{l}\text { HCM } \\
(n=112)\end{array}$ & $\begin{array}{l}\text { Myocarditis } \\
(n=17)\end{array}$ & $\begin{array}{l}\text { CAD } \\
(n=293)\end{array}$ \\
\hline 1 & Native T1 & $11(100 \%)$ & $88(56.1 \%)$ & $60(53.6 \%)$ & $7(41.2 \%)$ & $90(30.7 \%)$ \\
\hline 2 & ECV synthetic & $9(81.8 \%)$ & $114(72.6 \%)$ & $52(46.4 \%)$ & $7(41.2 \%)$ & 158 (53.9\%) \\
\hline 3 & $\mathrm{~T} 1$ and ECV synthetic & $11(100.0 \%)$ & $125(79.6 \%)$ & $60(53.6 \%)$ & $7(41.2 \%)$ & $175(59.7 \%)$ \\
\hline 1 vs. 3 & P-value & - & $<0.001$ & 1.000 & 1.000 & $<0.001$ \\
\hline 2 vs. 3 & P-value & - & 0.001 & 0.229 & 1.000 & $<0.001$ \\
\hline
\end{tabular}

in native $\mathrm{T} 1$ among patients with light chain $(\mathrm{AL})$ cardiac amyloidosis that had definite cardiac involvement by endomyocardial biopsy, and in AL cardiac amyloidosis patients with uncertain or absent cardiac involvement by echocardiogram. Native T1 CMR was observed to be more sensitive than other methods for detecting cardiac amyloidosis [26]. The diagnosis of cardiac amyloidosis by using LGE had many limitations. First, LGE in patients with cardiac amyloidosis can have many patterns. It can be atypical or patchy especially in patients with early 

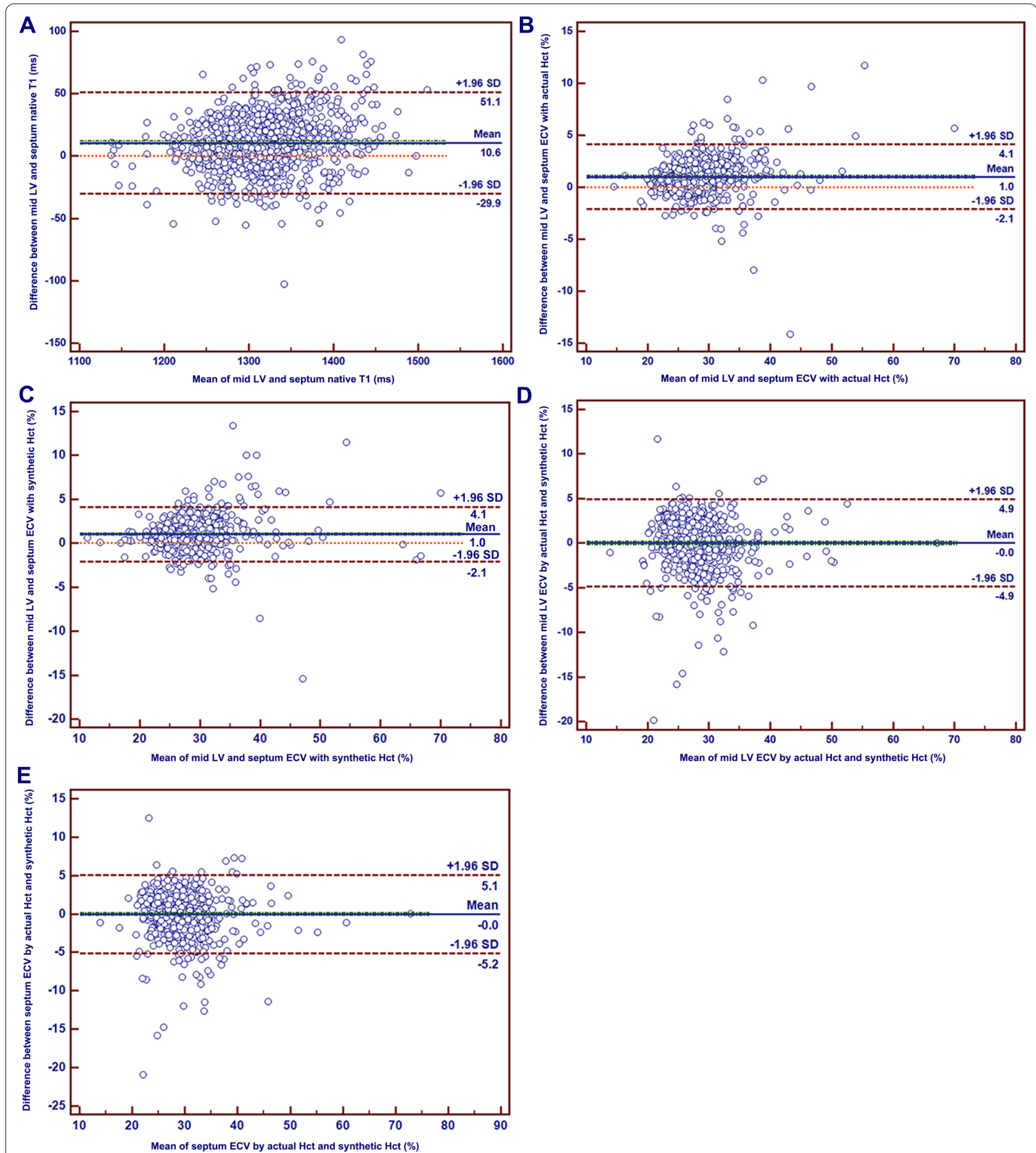

Fig. 6 Bland-Altman comparison of different methods for measuring native T1 and ECV. A mid LV and septum native T1, B mid LV and septum ECV with actual Hct, $\mathbf{C}$ mid LV and septum ECV with synthetic Hct, $\mathbf{D}$ mid LV ECV by actual Hct and synthetic Hct, $\mathbf{E}$ septum ECV by actual Hct and synthetic Hct

disease [27]. Second, amyloid deposition in the interstitium may reduce the contrast between blood and myocardium and the two components may null together. Thus, myocardium may appear normal in LGE images even the whole myocardium is diseased. The results of our study demonstrated that native $\mathrm{T}$ mapping and $\mathrm{ECV}$ are very 
accurate for the detection of cardiac amyloidosis. Native T1 mapping also have benefit in the detection of disease in case of impaired renal function that prohibit the use of CMR contrast agents and cannot acquire LGE images. Besides, T1 and ECV changes can provide earlier disease detection, even before LGE is detected [28].

In the present study, native T1 mapping was found to be an effective test for differentiating between controls and DCM with good diagnostic accuracy, specificity, and NPV, but not for sensitivity and PPV. The possible reason for limited sensitivity and PPV may be related to the accuracy of co-registration of thin-wall myocardium in DCM cases that is subjected to a potential measurement errors [29]. For differentiating HCM from controls, the average native $\mathrm{T} 1$ was found to have good diagnostic accuracy, specificity, and NPV, but it has limited sensitivity and PPV. Goebel, et al. reported significant difference in subgroup analysis of the average native T1 value between healthy heart patients and DCM patients $(p<0.001)$, and between healthy heart patients and HCM patients $(p=0.035)$. The AUC for the DCM group was $0.814(p<0.001)$, and the AUC for the HCM group was $0.688(p=0.067)[12]$ which is similar to the AUC results of our study.

Myocarditis patients usually have nonspecific signs, symptoms, and laboratory findings. Myocarditis is often diagnosed by exclusion of other cardiac causes. The diagnosis of myocarditis by CMR often requires multiple techniques, including early gadolinium enhancement, T2W, and LGE imaging. Updated recommendations of CMR criteria using novel CMR techniques, such as T1 mapping, T2 mapping, T2W image, and ECV, have more diagnostic accuracy [30]. The present study showed that native $\mathrm{T} 1$ mapping can differentiate myocarditis from controls with high accuracy, with an AUC of $0.710(p=0.003)$ for mid LV native T1, and with an AUC of $0.752(p<0.001)$ for septum native T1. Both ROI approaches showed high specificity and NPV, but limited sensitivity and PPV. However, we were not able to determine if native $\mathrm{T} 1$ mapping could distinguish between acute and convalescent myocarditis due to the small sample size in the acute myocarditis group $(n=2)$. Hinojar, et al., reported native $\mathrm{T} 1$ to be an independent discriminator between healthy patients and myocarditis patients, as well as for differentiating between acute and convalescent stage of myocarditis with high diagnostic accuracy, PPV, and NPV. The mean native T1 value was found to be higher in both the acute and convalescent myocarditis groups than in the healthy patient group [31]. The difference in results of Hinojar et al. and our study may be due to the smaller sample size of myocarditis in our study and may be related to the influence of the amount and location of LGE in myocarditis cases on T1 and ECV measurement.

In the CAD group, native T1 mapping test showed statistical significance in remote myocardium (excluding scarred segment) for mid LV short-axis approach and septal approach. The present study's CMR imaging analysis protocol for the CAD group excluded subdivision segment with scar for native T1 analysis since the scar area since the scar would markedly increase native T1. However, lipomatous changes within the scar may decrease native T1 [32]. Previous studies showed inconsistent results on native $\mathrm{T} 1$ in remote myocardium of $\mathrm{CAD}$ cases. Some studies showed that native T1 of remote myocardium are similar to controls [12, 33, 34] but some studies showed an increased native $\mathrm{T} 1$ in remote myocardium [13]. Some patients might have a diffuse disease without a dense scar in the remote area and some may have ischemic myocardium which also can increase native $\mathrm{T} 1$ values [35].

Native T1 myocardium has acquisition without gadolinium-based contrast agent, which is an important advantage for patients with significant renal impairment who may be at increased risk for nephrogenic systemic sclerosis. The calculated ECV from T1 mapping reduces the variability of test results because of calculation from the ratio of change in myocardial $\mathrm{T} 1$ relative to blood pool T1 pre- and post-contrast [36]. The advantage of ECV is that it reduces systematic errors in technique, and results in less variability at different field strengths and across different vendor platforms [37]. The higher mean mid LV ECV value in the amyloidosis, DCM, HCM, and CAD groups demonstrated statistically significant difference from controls, similar to average native T1, except the same significant ability to differentiate from controls was not observed in the myocarditis subgroup. The mean ECV of myocarditis showed statistical significance when ECV was calculated using synthetic Hct. The observed differences in statistical significance relative to ECV between actual Hct and synthetic Hct in this study can be explained by the small number of patients and the lower number of patients in the actual Hct group.

The value of calculated ECV using actual Hct and synthetic Hct in each group showed concordant results with high degree of agreement which supported the report of the synthetic Hct formula by Fent, et al., [7].

\section{Study limitations}

This study has some mentionable limitations. Firstly, no healthy volunteer data were included in this study. Patients having clinical indication for CMR without evidence of ischemia or infarction was used as the control group. It has been shown that patients with suspected 
CAD and negative adenosine CMR had an excellent prognosis with no major adverse cardiac event during follow up [38]. Results of meta-analysis also demonstrated a very excellent prognosis in patients with negative stress CMR study [39, 40]. Besides, in order to acquire ECV, post contrast T1 is needed for calculation. The injection of contrast agent in healthy volunteers is not practical in a clinical setup and it is difficult to obtain the ethical approval. A previous study that reported reference values of T1 in 'normal healthy myocardium' also used patients who were referred for CMR with negative CMR finding to define the reference value of T1 in normal [41]. Another study also used patients who were referred for CMR with normal CMR findings as control and compared to cardiovascular diseases [13]. They also mentioned that patients with negative CMR findings had $\mathrm{T} 1$ values similar to data of healthy volunteer from previous report [13]. The study objectives were designed to seek for the difference of $\mathrm{T} 1$ and $\mathrm{ECV}$ values among various groups of patients with myocardial disease and CAD in clinical practice rather than to distinguish $\mathrm{T} 1$ and $\mathrm{ECV}$ values between healthy volunteers and patients. The results of our study may be a more practical approach in real-world practice. Second, due to the retrospective study design, we used actual Hct within 6 months prior to CMR date to calculate ECV. Although we realized that certain factor such as hydration status could have effect on $\mathrm{T} 1$ relaxation time [42], we considered a 6-month period to be an acceptable duration. Furthermore, to reduce this limitation, we reported the calculated ECV with both actual Hct values and synthetic Hct values which were derived from $\mathrm{T} 1$ measurement of blood pool in the same sequence that myocardial $\mathrm{T} 1$ values were acquired. The Bland-Altman plot confirmed good agreement between the two methods, which supports that synthetic Hctderived ECV may be used in place of conventional ECV in the cases that have missing Hct data. Third, native T1 mapping was performed in one mid LV short-axis slice, not the entire myocardial region. However, mid LV slice is recommended by standard guideline [6] and it should reflect pathology in patients with diffuse disease. Fourth, the number of patients in some subgroups of myocardial disease were rather small, so we could not test for differentiation between acute and convalescent myocarditis. Fifth, although we performed routine T1 mapping in the study population, we used the conventional criteria for the diagnosis of disease. Sixth, among patients in CAD group, this study aimed to measure the average native $\mathrm{T} 1$ and $\mathrm{ECV}$ of remote myocardium of CAD, not for infarcted area. Lastly, T1 mapping and ECV is well known to be sequence and technique dependent. This may limit the generalizability of the methodology. However, myocardial T1 mapping is a powerful clinical tool for soft tissue characteristic classification in spite of the absence of established reference values.

There were some strengths of this study. First, we reported data on a large number of patients. Second, we are the one of the few studies [24] that reported results from 3.0 Tesla CMR. Third, we compared results of myocardial disease and controls, and CAD versus controls. Fourth, we studied T1 mapping from ROI of mid LV slice and mid LV septum, and we used ECV from actual Hct and synthetic Hct.

\section{Conclusions}

Although native $\mathrm{T} 1$ and ECV of patients with cardiomyopathy and CAD were significantly higher than controls, the values overlapped. The greatest clinical utilization was found for the amyloidosis group.

\section{Abbreviations}

CAD: Coronary artery disease; CMR: Cardiac magnetic resonance; DCM: Dilated cardiomyopathy; ECV: Extracellular volume fraction; HCM: Hypertrophic cardiomyopathy; Hct: Hematocrit; LGE: Late gadolinium enhancement; LV: Left ventricle; NPV: Negative predictive value; PPV: Positive predictive value; ROC: Receiver operating characteristics; ROI: Region of interest.

\section{Supplementary Information}

The online version contains supplementary material available at https://doi. org/10.1186/s12872-021-02086-3.

Additional file 1. Cutoff values from receiver operating characteristic (ROC) curve analysis of native T1 in myocardial disease and CAD Case: control $(1: 1)$

Additional file 2. Cutoff values from receiver operating characteristic (ROC) curve analysis of mid LV short-axis ECV in myocardial disease and CAD (1:1).

\section{Acknowledgements \\ The authors thank Ahthit Yindeengam for statistical analysis and data management.}

\section{Authors' contributions}

RT, RK: concept and design, data acquisition, interpretation of data, manuscript preparation, manuscript revision, and manuscript review. TS: manuscript revision, and manuscript review. PT: data acquisition, interpretation of data. All authors have read and agreed to the published version of the manuscript, and approved the submission of this manuscript for journal publication.

\section{Funding \\ None.}

\section{Availability of data and materials}

The dataset that was used to support the results and conclusion of this study are included within the manuscript. Additional data are available upon contacting Rungroj Krittayaphong at rungroj.kri@mahidol.ac.th with the reasonable request. 


\section{Declarations}

\section{Ethics approval and consent to participate}

This study was approved by the Siriraj Institutional Review Board (SIRB) of the Faculty of Medicine Siriraj Hospital, Mahidol University, Bangkok, Thailand. According to committee of SIRB, consent to participate was not required due to the retrospective nature of this study. The study was conducted in compliance with the principles of the Declaration of Helsinki, International Conference on Harmonization Good Clinical Practice guidelines on noninterventional studies.

\section{Consent for publication}

Not applicable.

\section{Competing interests}

The authors declare that they have no competing interests.

\section{Author details}

'Division of Cardiology, Department of Medicine, Faculty of Medicine Siriraj Hospital, Mahidol University, 2 Wanglang Road, Bangkoknoi, Bangkok 10700, Thailand. ${ }^{2}$ Her Majesty Cardiac Center, Faculty of Medicine Siriraj Hospital, Mahidol University, Bangkok, Thailand.

Received: 14 December 2020 Accepted: 25 May 2021 Published online: 03 June 2021

\section{References}

1. Hinderer S, Schenke-Layland K. Cardiac fibrosis: a short review of causes and therapeutic strategies. Adv Drug Deliv Rev. 2019;146:77-82.

2. Bulluck H, Maestrini V, Rosmini S, Abdel-Gadir A, Treibel TA, Castelletti S, et al. Myocardial T1 mapping. Circ J. 2015;79:487-94.

3. Moon JC, Messroghli DR, Kellman P, Piechnik SK, Robson MD, Ugander M, et al. Myocardial T1 mapping and extracellular volume quantification: a Society for Cardiovascular Magnetic Resonance (SCMR) and CMR Working Group of the European Society of Cardiology consensus statement. J Cardiovasc Magn Reson. 2013;15:92.

4. Bull S, White SK, Piechnik SK, Flett AS, Ferreira VM, Loudon M, et al. Human non-contrast T1 values and correlation with histology in diffuse fibrosis. Heart. 2013;99:932-7.

5. Haaf P, Garg P, Messroghli DR, Broadbent DA, Greenwood JP, Plein S Cardiac T1 Mapping and Extracellular Volume (ECV) in clinical practice: a comprehensive review. J Cardiovasc Magn Reson. 2016;18:89.

6. Messroghli DR, Moon JC, Ferreira VM, Grosse-Wortmann L, He T, Kellman $P$, et al. Clinical recommendations for cardiovascular magnetic resonance mapping of $\mathrm{T} 1, \mathrm{~T} 2, \mathrm{~T}_{2}^{*}$ and extracellular volume: A consensus statement by the Society for Cardiovascular Magnetic Resonance (SCMR) endorsed by the European Association for Cardiovascular Imaging (EACVI). J Cardiovasc Magn Reson. 2017:19:75.

7. Fent GJ, Garg P, Foley JRJ, Swoboda PP, Dobson LE, Erhayiem B, et al. Synthetic myocardial extracellular volume fraction. JACC Cardiovasc Imaging. 2017;10:1402-4

8. Puntmann VO, Carr-White G, Jabbour A, Yu CY, Gebker R, Kelle S, et al. T1-mapping and outcome in nonischemic cardiomyopathy: all-cause mortality and heart failure. JACC Cardiovasc Imaging. 2016;9:40-50.

9. Duca F, Kammerlander AA, Zotter-Tufaro C, Aschauer S, Schwaiger ML, Marzluf BA, et al. Interstitial fibrosis, functional status, and outcomes in heart failure with preserved ejection fraction: insights from a prospective cardiac magnetic resonance imaging study. Circ Cardiovasc Imaging. 2016;9.

10. Zhuang B, Sirajuddin A, Wang S, Arai A, Zhao S, Lu M. Prognostic value of T1 mapping and extracellular volume fraction in cardiovascular disease: a systematic review and meta-analysis. Heart Fail Rev. 2018;23:723-31.

11. Puntmann VO, Peker $E$, Chandrashekhar Y, Nagel E. T1 mapping in characterizing myocardial disease: a comprehensive review. Circ Res. 2016;119:277-99.

12. Goebel J, Seifert I, Nensa F, Schemuth HP, Maderwald S, Quick HH, et al. Can native T1 mapping differentiate between healthy and diffuse diseased myocardium in clinical routine cardiac MR imaging? PLoS ONE. 2016;11:e0155591.

13. Liu JM, Liu A, Leal J, McMillan F, Francis J, Greiser A, et al. Measurement of myocardial native T1 in cardiovascular diseases and norm in 1291 subjects. J Cardiovasc Magn Reson. 2017;19:74.

14. Maceira AM, Joshi J, Prasad SK, Moon JC, Perugini E, Harding I, et al. Cardiovascular magnetic resonance in cardiac amyloidosis. Circulation. 2005; 111:186-93

15. Japp AG, Gulati A, Cook SA, Cowie MR, Prasad SK. The diagnosis and evaluation of dilated cardiomyopathy. J Am Coll Cardiol. 2016;67:2996-3010.

16. Elliott PM, Anastasakis A, Borger MA, Borggrefe M, Cecchi F, Charron P, et al. 2014 ESC Guidelines on diagnosis and management of hypertrophic cardiomyopathy: the Task Force for the Diagnosis and Management of Hypertrophic Cardiomyopathy of the European Society of Cardiology (ESC). Eur Heart J. 2014;35:2733-79.

17. Friedrich MG, Sechtem U, Schulz-Menger J, Holmvang G, Alakija P, Cooper $\mathrm{LT}$, et al. Cardiovascular magnetic resonance in myocarditis: a JACC White Paper. J Am Coll Cardiol. 2009:53:1475-87.

18. Dabir D, Vollbrecht TM, Luetkens JA, Kuetting DLR, Isaak A, Feisst A, et al. Multiparametric cardiovascular magnetic resonance imaging in acute myocarditis: a comparison of different measurement approaches. J Cardiovasc Magn Reson. 2019;21:54.

19. Antiochos P, Ge Y, Steel K, Chen YY, Bingham S, Abdullah S, et al. Evaluation of stress cardiac magnetic resonance imaging in risk reclassification of patients with suspected coronary artery disease. JAMA Cardiol. 2020

20. Higgins DM, Moon JC. Review of T1 mapping methods: comparative effectiveness including reproducibility issues. Curr Cardiovasc Imaging Rep. 2014;7:9252.

21. Cerqueira MD, Weissman NJ, Dilsizian V, Jacobs AK, Kaul S, Laskey WK, et al. Standardized myocardial segmentation and nomenclature for tomographic imaging of the heart. A statement for healthcare professionals from the Cardiac Imaging Committee of the Council on Clinical Cardiology of the American Heart Association. Int J Cardiovasc Imaging. 2002;18:539-42.

22. Xue H, Shah S, Greiser A, Guetter C, Littmann A, Jolly MP, et al. Motion correction for myocardial T1 mapping using image registration with synthetic image estimation. Magn Reson Med. 2012;67:1644-55.

23. Tilborghs S, Dresselaers T, Claus P, Claessen G, Bogaert J, Maes F, et al. Robust motion correction for cardiac T1 and ECV mapping using a T1 relaxation model approach. Med Image Anal. 2019:52:212-27.

24. Puntmann VO, Voigt T, Chen Z, Mayr M, Karim R, Rhode K, et al. Native T mapping in differentiation of normal myocardium from diffuse disease in hypertrophic and dilated cardiomyopathy. JACC Cardiovasc Imaging. 2013;6:475-84

25. h-Ici DO, Jeuthe S, Al-Wakeel N, Berger F, Kuehne T, Kozerke S, et al. T1 mapping in ischaemic heart disease. Eur Heart J Cardiovasc Imaging. 2014:15:597-602

26. Karamitsos TD, Piechnik SK, Banypersad SM, Fontana M, Ntusi NB, Ferreira $\mathrm{VM}$, et al. Noncontrast T1 mapping for the diagnosis of cardiac amyloidosis. JACC Cardiovasc Imaging. 2013;6:488-97.

27. Di Bella G, Minutoli F, Mazzeo A, Vita G, Oreto G, Carerj S, et al. MRI of cardiac involvement in transthyretin familial amyloid polyneuropathy. AJR Am J Roentgenol. 2010;195:W394-399.

28. Banypersad SM. The evolving role of cardiovascular magnetic resonance imaging in the evaluation of systemic amyloidosis. Magn Reson Insights. 2019:12:1178623 × 19843519.

29. Reiter U, Reiter C, Krauter C, Fuchsjager M, Reiter G. Cardiac magnetic resonance T1 mapping. Part 2: Diagnostic potential and applications. Eur J Radiol. 2018;109:235-47.

30. Ferreira VM, Schulz-Menger J, Holmvang G, Kramer CM, Carbone I, Sechtem $U$, et al. Cardiovascular magnetic resonance in nonischemic myocardial inflammation: expert recommendations. J Am Coll Cardiol. 2018;72:3158-76

31. Hinojar R, Foote L, Arroyo Ucar E, Jackson T, Jabbour A, Yu CY, et al. Native $\mathrm{T} 1$ in discrimination of acute and convalescent stages in patients with clinical diagnosis of myocarditis: a proposed diagnostic algorithm using CMR. JACC Cardiovasc Imaging. 2015;8:37-46.

32. Kellman P, Bandettini WP, Mancini C, Hammer-Hansen S, Hansen MS, Arai AE. Characterization of myocardial T1-mapping bias caused by 
intramyocardial fat in inversion recovery and saturation recovery techniques. J Cardiovasc Magn Reson. 2015;17:33.

33. Liu A, Wijesurendra RS, Liu JM, Greiser A, Jerosch-Herold M, Forfar JC, et al. Gadolinium-free cardiac MR stress T1-mapping to distinguish epicardial from microvascular coronary disease. J Am Coll Cardiol. 2018;71:957-68,

34. White SK, Sado DM, Fontana M, Banypersad SM, Maestrini V, Flett AS, et al. T1 mapping for myocardial extracellular volume measurement by CMR: bolus only versus primed infusion technique. JACC Cardiovasc Imaging. 2013:6:955-62.

35. Liu A, Wijesurendra RS, Francis JM, Robson MD, Neubauer S, Piechnik SK et al. Adenosine stress and rest $\mathrm{T} 1$ mapping can differentiate between ischemic, infarcted, remote, and normal myocardium without the need for gadolinium contrast agents. JACC Cardiovasc Imaging. 2016;9:27-36

36. McDiarmid AK, Broadbent DA, Higgins DM, Swoboda PP, Kidambi A, Ripley DP, et al. The effect of changes to MOLLI scheme on T1 mapping and extra cellular volume calculation in healthy volunteers with 3 tesla cardiovascular magnetic resonance imaging. Quant Imaging Med Surg. 2015;5:503-10.

37. Jellis CL, Kwon DH. Myocardial T1 mapping: modalities and clinical applications. Cardiovasc Diagn Ther. 2014:4:126-37.

38. Ingkanisorn WP, Kwong RY, Bohme NS, Geller NL, Rhoads KL, Dyke CK, et al. Prognosis of negative adenosine stress magnetic resonance in patients presenting to an emergency department with chest pain. J Am Coll Cardiol. 2006;47:1427-32
39. Lipinski MJ, McVey CM, Berger JS, Kramer CM, Salerno M. Prognostic value of stress cardiac magnetic resonance imaging in patients with known or suspected coronary artery disease: a systematic review and meta-analysis. J Am Coll Cardiol. 2013;62:826-38.

40. Smulders MW, Jaarsma C, Nelemans PJ, Bekkers S, Bucerius J, Leiner T, et al. Comparison of the prognostic value of negative non-invasive cardiac investigations in patients with suspected or known coronary artery disease-a meta-analysis. Eur Heart J Cardiovasc Imaging. 2017;18:980-7.

41. Dabir D, Child N, Kalra A, Rogers T, Gebker R, Jabbour A, et al. Reference values for healthy human myocardium using a T1 mapping methodology: results from the International T1 Multicenter cardiovascular magnetic resonance study. J Cardiovasc Magn Reson. 2014;16:69.

42. Luetkens JA, Voigt M, Faron A, Isaak A, Mesropyan N, Dabir D, et al. Influence of hydration status on cardiovascular magnetic resonance myocardial T1 and T2 relaxation time assessment: an intraindividual study in healthy subjects. J Cardiovasc Magn Reson. 2020;22:63.

\section{Publisher's Note}

Springer Nature remains neutral with regard to jurisdictional claims in published maps and institutional affiliations.
Ready to submit your research? Choose BMC and benefit from:

- fast, convenient online submission

- thorough peer review by experienced researchers in your field

- rapid publication on acceptance

- support for research data, including large and complex data types

- gold Open Access which fosters wider collaboration and increased citations

- maximum visibility for your research: over 100M website views per year

At BMC, research is always in progress.

Learn more biomedcentral.com/submissions 